\title{
Changes of Parvalbumin Expression in the Spinal Cord after Peripheral Inflammation
}

\author{
G. ZACHAROVA, D. SOJKA, J. PALECEK
}

Department of Functional Morphology, Institute of Physiology, Academy of Sciences of the Czech Republic, Prague, Czech Republic

Received January 24, 2008

Accepted May 14, 2008

On-line July 18, 2008

\section{Summary}

Parvalbumin (PV) is a calcium-binding protein that is expressed by numerous neuronal subpopulations in the central nervous system. Staining for PV was often used in neuroanatomical studies in the past. Recently, several studies have suggested that PV acts in neurons as a mobile endogenous calcium buffer that affects temporo-spatial characteristics of calcium transients and is involved in modulation of synaptic transmission. In our experiments, expression of $\mathrm{PV}$ in the lumbar dorsal horn spinal cord was evaluated using densitometric analysis of immunohistological sections and Western-blot techniques in control and arthritic rats. There was a significant reduction of PV immunoreactivity in the superficial dorsal horn region ipsilateral to the arthritis after induction of the peripheral inflammation. The ipsilateral area and intensity of PV staining in this area were reduced to $38 \%$ and $37 \%$, respectively, out of the total PV staining on both sides. It is suggested that this reduction may reflect decreased expression of PV in GABAergic inhibitory neurons. Reduction of PV concentration in the presynaptic GABAergic terminals could lead to potentiation of inhibitory transmission in the spinal cord. Our results suggest that changes in expression of calcium-binding proteins in spinal cord dorsal horn neurons may modulate nociceptive transmission.

\section{Key words}

Arthritis • Calcium buffer • Hyperalgesia • GABA • Parvalbumin

\section{Corresponding author}

Jiri Palecek M.D., Ph.D., Department of Functional Morphology, Institute of Physiology ASCR, Videnska 1083, 14220 Praha 4, Czech Republic. Fax: +420 241062488.

E-mail: palecek@biomed.cas.cz

\section{Introduction}

Numerous proteins and receptors were shown to play an important role in modulation of nociceptive signaling at spinal cord level. Parvalbumin (PV) is a calcium-binding protein (CBP) that has often been used as a marker of specific neuronal populations in neuroanatomical and immunohistological studies in the past. Recently, several studies have considered the biophysical consequences of the presence of PV in the cells as a calcium buffer (Neher and Augustine 1992, Zhou and Neher 1993). Synaptic activity as well as number of other cellular processes are regulated by local and/or global changes of intracellular calcium level. During propagation of action potentials, intracellular calcium level is increased mainly due to calcium influx through voltage-dependent calcium channels. Other important sources of extracellular calcium in the dendrites during synaptic activity are calcium-permeable NMDA and AMPA channels. The intracellular calcium concentration is further regulated by several processes, such as calcium uptake into intracellular stores, extrusion mechanisms and endogenous calcium buffering (Berridge et al. 2003).

Calcium-binding proteins such as parvalbumin, calbindin and others represent mobile endogenous buffers that can substantially affect the temporo-spatial characteristics of any intracellular calcium concentration change, depending on their respective biophysical calcium-binding characteristics. It was shown in other neuronal systems that a low level of CBP leads to high amplitude short-lasting calcium concentration changes, while the presence of a high level of calcium buffers in the cell leads to low amplitude, longer duration calcium 


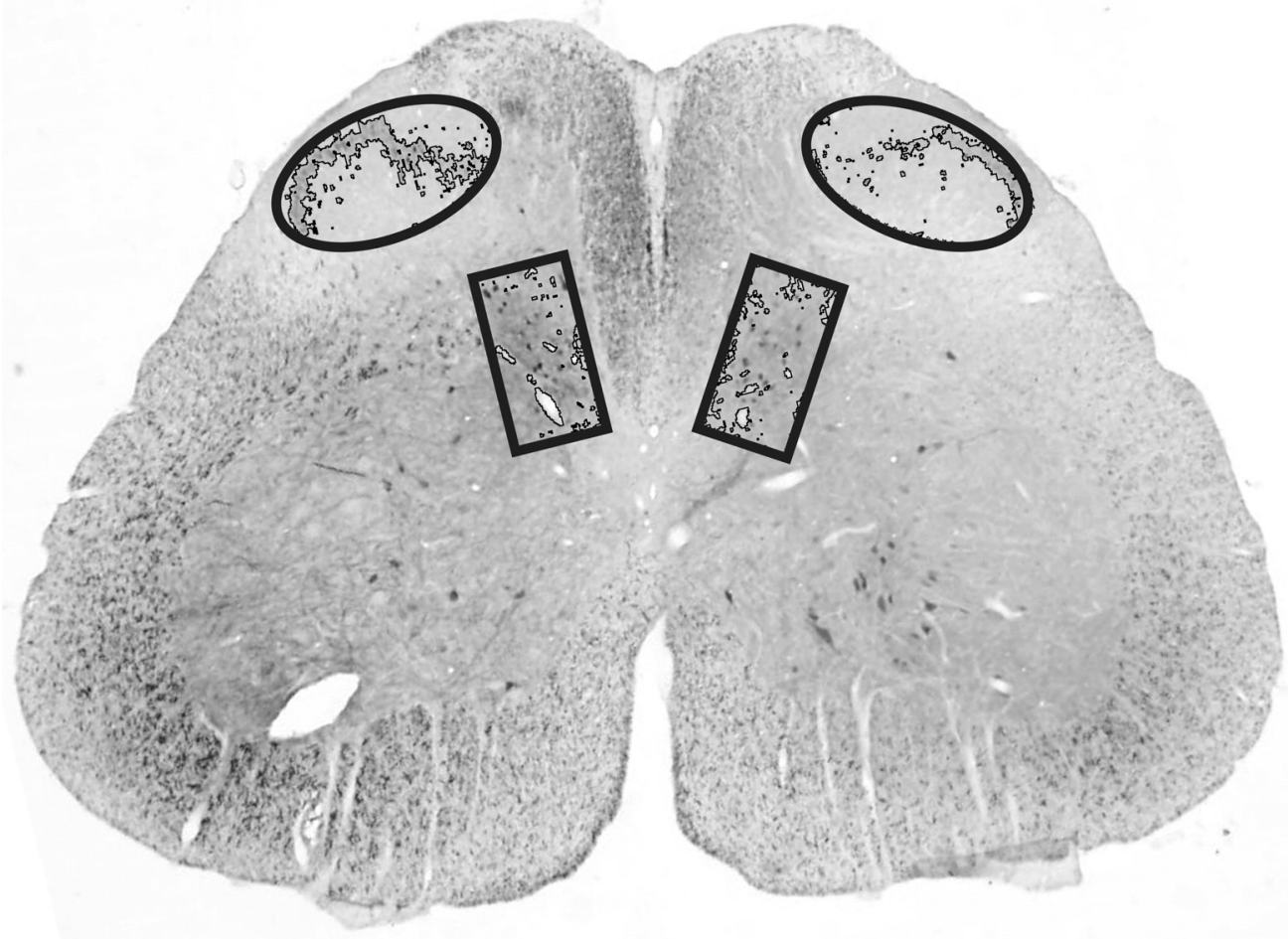

Fig. 1. Off-line densitometric analysis of L5 spinal cord segments performed using an AIDA software package. Two symmetrical areas in the dorsal horn and ventromedial region were used for the densitometric analysis.

transients under the same circumstances (Chard et al. 1993, Palecek et al. 1999). While both fast (calbindin) and slow (PV) calcium buffers can reduce the calcium transient peak, PV selectively increases the fast component of the calcium transient decay (Chard et al. 1993, Caillard et al. 2000, Lee et al. 2000, Schmidt et al. 2003, Muller et al. 2007).

It is accepted that in some chronic pain states associated with the presence of pathologically increased sensitivity such as allodynia and hyperalgesia, central sensitization due to synaptic modulation at the spinal cord level may play an important role (Woolf and Thompson 1991, Willis 2001, Spicarova and Palecek 2008). It was previously suggested that this process is calciumdependent (Willis 2001, see also Malenka and Nicoll 1999). The presence or absence of CBPs acting as calcium buffers in the pre- or postsynaptic part of the synapse could thus effectively modulate synaptic transmission at the spinal cord level. Studies of PV distribution in the lumbar spinal cord have shown that it is mostly located in a strongly immunopositive band of small neuronal cell bodies and neuropil in the inner part of lamina II (Antal et al. 1990, Ren and Ruda 1994) and at the border of laminae II/III (Yamamoto et al.1989). There is also strong PV immunostaining present in the neck of the spinal cord grey matter in the ventromedial region (lamina VII). Numerous PV-positive neurons are present throughout the deeper laminae and also in the ventral horn (Ren and Ruda 1994).

In this study the changes of PV expression in the lumbar spinal cord dorsal horn after experimentally induced peripheral inflammation were observed in rats. The level of PV expression was assessed by densitometry in the superficial dorsal horn and in the ventromedial part of the spinal cord on immunohistochemically stained sections from lumbar segment L5. Further confirmation of the changes in PV expression after peripheral inflammation was provided by Western blot analysis of the spinal cord tissue.

\section{Methods}

All procedures used in these experiments were reviewed and approved by the Institutional Animal Care and Use Committee and were consistent with the guidelines of the International Association for the Study of Pain for the care and use of laboratory animals. Adult male Wistar rats (250-350 g) were kept in plastic cages with soft bedding, free access to food and water and were maintained on a $12 \mathrm{~h}$ light, $12 \mathrm{~h}$ dark cycle. In total, 16 animals were used in this study.

Experimental arthritis was induced by unilateral 


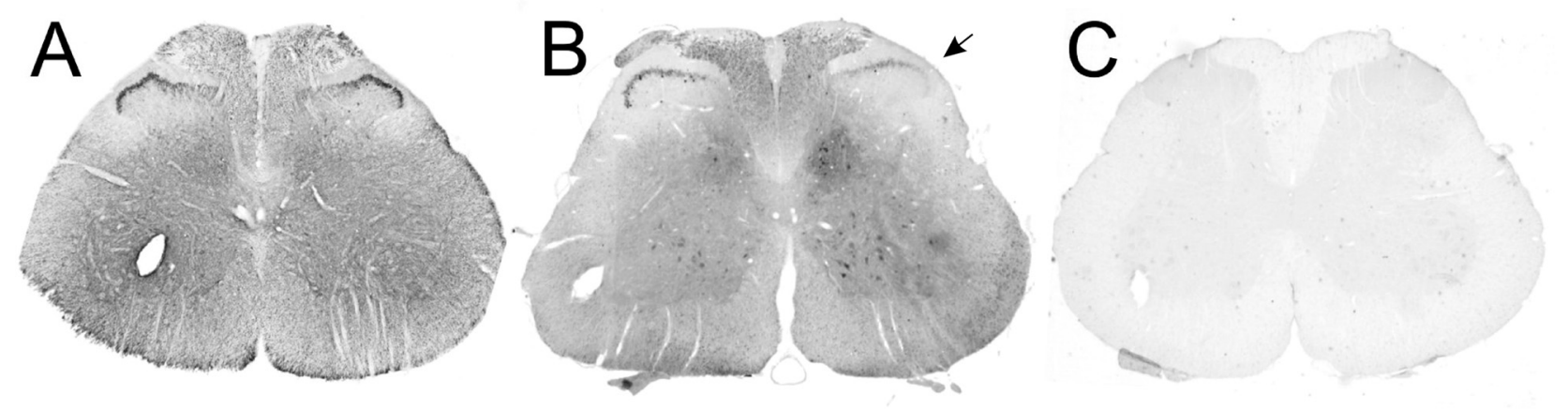

Fig. 2. Examples of immunostaining for $P V$ in the lumbar spinal cord. (A) There was no side to side change of the PV staining in the control animals. (B) There was a marked reduction of the staining in the dorsal horn ipsilateral to the inflammation (arrow) in a spinal cord slice from the experimental animal. (C) No staining was present when primary antibody was omitted as a control.

intra-articular knee injection of a $3 \%$ mixture of kaolin and carrageenan in saline solution under ether anesthesia. The animals were left to recover in their home cages. Animals were deeply anesthetized with sodium pentobarbital ( $80 \mathrm{mg} / \mathrm{kg}$ intraperitoneally) $28 \mathrm{~h}$ later and perfused transcardially with heparinized saline $(0.9 \%$ $\mathrm{NaCl}$ ) followed by $500 \mathrm{ml}$ of ice cold $4 \%$ paraformaldehyde in $0.1 \mathrm{M}$ phosphate buffer. Spinal cord tissue was removed after the perfusion, the control side marked with a pin, and post-fixed overnight in the same fixative and cryoprotected $24 \mathrm{~h}$ in $30 \%$ sucrose solution in phosphate buffer (PB). Transverse cryostat sections $(30 \mu \mathrm{m})$ from the L5 spinal cord segment were obtained from five experimental and three intact control rats. Every third slice was collected and immunostained for PV by the streptavidin-biotin-peroxidase complex method. First, the sections were washed with a solution of $0.1 \mathrm{M}$ phosphate-buffered-saline (PBS) three times for $10 \mathrm{~min}$ and then incubated in blocking solution of $3 \%$ normal donkey serum (NDS) for $30 \mathrm{~min}$ at room temperature, followed by overnight incubation at $4{ }^{\circ} \mathrm{C}$ in primary antibody solution (mouse monoclonal antiparvalbumin; Sigma). After incubation, tissue sections were washed in $1 \%$ NDS three times for $10 \mathrm{~min}$ and incubated for $2 \mathrm{~h}$ at room temperature in biotinylated secondary antibody solution (1:400 for $2 \mathrm{~h}$ biotin-SPconjugated donkey anti-mouse IgG; Jackson Immuno Research Laboratories, Inc., USA). Then the sections were washed in $\mathrm{PB}$ twice for $10 \mathrm{~min}$ and incubated in peroxidase-conjugated streptavidin (dilution 1:600, Jackson Immuno Research) for $2 \mathrm{~h}$ at room temperature. The reaction product was visualized with $0.01 \%$ hydrogen peroxide and $0.05 \%$ diaminobenzidine as the chromogen. After rinsing in $0.1 \mathrm{M}$ PB for $10 \mathrm{~min}$, the sections were mounted onto gelatinized slides and coverslipped. The whole spinal cord section was photographed with an Olympus BX-80 and 5Mpix Digital camera in one image. Off-line densitometric analysis of the spinal cord images was performed using the AIDA software package. Two symmetrical areas on each side in the superficial dorsal horn and in the ventromedial part were selected for analysis (Fig. 1). A small area in the part of the tissue without positive signal was selected to determine the threshold value for the immunopositive signal intensity. The software calculated the area of positivity within the preselected region, the sum of intensity in these areas, and the quotient of these values in absolute values and in percent. The percent values on a given side of the analyzed section were calculated as the percentage of immunopositive area or intensity, compared to the sum of positive areas or intensities on both sides. Percent values were used for statistical analysis to account for the differences between animals. The differences in the intensity of immunostaining in a side-to-side comparison were evaluated using paired t-tests.

For further confirmation of PV expression levels, SDS-PAGE and Western blotting were used in another group of animals. Rats were anesthetized with sodium pentobarbital $(80 \mathrm{mg} / \mathrm{kg}$ ip) and laminectomy was performed. Dissected lumbar spinal cord was rapidly immersed in ice-cold physiological solution and hemisected. Left and right side spinal cord parts (L3-5) were weighed and homogenized with a hand-held pellet pestle in $30 \mathrm{mM}$ Tris- $\mathrm{HCl}$ buffer (w/v 1:10) containing $0.1 \mu \mathrm{l}$ of an inhibitor of proteases (Sigma P8340) per $1 \mathrm{mg}$ of tissue. Prepared samples were left on the ice for $10 \mathrm{~min}$ and centrifuged at $5000 \mathrm{rpm}$ for $10 \mathrm{~min}$ at $4{ }^{\circ} \mathrm{C}$. Supernatant was removed and centrifuged again at $13000 \mathrm{rpm}$ for $20 \mathrm{~min}$. An aliquot was taken for 
spectrophotometric total protein quantification using an Eppendorf BioPhotometer (Eppendorf, Germany). The protein concentration for each sample was determined by the Bradford assay using a Bio-Rad Protein Assay kit according to the manufacturer's recommendations. For immunodetection of parvalbumin, protein samples and molecular mass standards (Bio-Rad) were resolved on $12 \%$ Tris/Tricine gels. Tris/Tricine SDS-PAGE was performed according to the method of Schägger and von Jagow (1991). Proteins were then transferred to a $0.2 \mu \mathrm{m}$ nitrocellulose membrane with blotting apparatus (Bio$\mathrm{Rad})$, at $15 \mathrm{~V}$ overnight. Western blots were developed as described by Towbin et al. (1979). Mouse monoclonal anti-parvalbumin (Sigma) was used as primary antibody at a dilution of 1:2000. The secondary antibody conjugated to horseradish peroxidase and/or biotinylated antibody followed by streptavidin peroxidase (Jackson Immuno Research Laboratories, Inc., USA) were used at a dilution of 1:6000, 1:32000 and 1:20000, respectively. The proteins were visualized with an ECL kit (Bio-Rad) and data were captured using Fuji LAS-1000 Imaging System CCD Camera. Quantitative analysis of immunoblots was performed using the image analysis program ImageJ (version $1.35 \mathrm{~h}$ ).
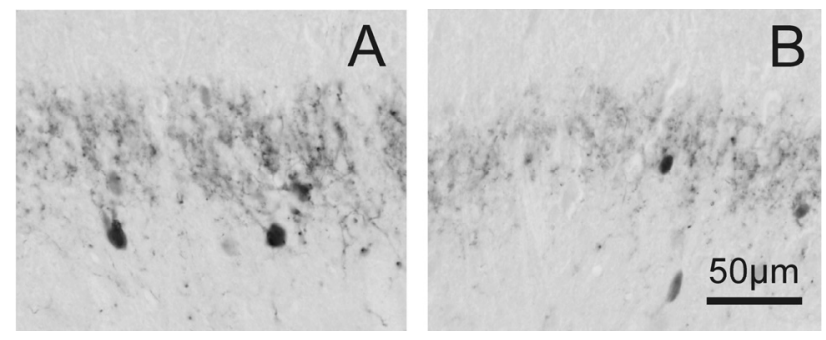

Fig. 3. Example of dorsal horn immunostaining for $\mathrm{PV}$ on the contralateral (A) and ipsilateral (B) side to the experimental arthritis. Most of the staining was present in the neuropil, while some stained cell bodies were also present.

\section{Results}

In this study the intensity of immunostaining for parvalbumin in ipsilateral and contralateral sides of the spinal cord in rats with unilateral experimental knee joint arthritis was analyzed. Two regions with high intensity immunostaining for PV within the dorsal horn were examined with densitometric analysis. One was in the superficial dorsal horn (substantia gelatinosa) and the second was in the ventromedial part of the dorsal horn. Five experimental and three control intact animals were used in the study. From each rat, 10 sections from lumbar segment L5 were analyzed.

Lower intensity and/or smaller area of positive parvalbumin staining in the substantia gelatinosa on the arthritic side was clearly evident in about $2 / 3$ of the sections examined (Fig. 2). The staining was present mostly in the neuropil with occasional immunopositive cell bodies, as can be seen on the higher magnification picture of the dorsal horn area (Fig. 3). The differences in the density and in the area of PV staining between the control side and the side ipsilateral to the induced arthritis were assessed by densitometric analysis. In the superficial dorsal horn, both area and the intensity of the PV immunostaining were confirmed to be significantly decreased on the side of the experimental arthritis when compared to the contralateral side (Fig. 4). The difference in the staining was even more significant when it was evaluated as an intensity/area quotient. In the ventromedial part of the dorsal horn no apparent difference was obvious. The densitometric analysis did not also reveal any significant difference in the area and intensity of the PV staining in the ventromedial part of the spinal cord. However, the quotient of the intensity/area was significantly reduced $(\mathrm{P}<0.05)$ on the side of the experimental arthritis (Fig. 4). In control rats, no significant difference was revealed by the densitometric measurement in any of the measured parameters when left and right sides of the spinal cord were compared in the same way as in the experimental animals. The percent values were 49.91 to $50.09 \%$ for the area and 49.82 to $50.18 \%$ for the intensity of the total in a left to right side comparison for the substantia gelatinosa region and 49.87 to $50.13 \%$ (area) and 49.81 to $50.19 \%$ (intensity) for the ventromedial region.

Western blot analysis of PV content in the spinal cord samples was used to confirm the densitometric immunohistochemical analysis (Fig. 5). Tissue from ipsilateral and contralateral sides of the spinal cord lumbar enlargement (L3-L5) in 5 animals was analyzed. The PV content in the spinal cord tissue ipsilateral to the arthritis was significantly reduced to $68 \pm 12 \%$ (SEM, $\mathrm{p}<0.05$, paired t-test) when compared to the control side. There was no significant difference in the PV content of the left and right sides in the control animals $(n=3)$.

\section{Discussion}

Our study demonstrated that peripheral inflammation can induce decreased expression of PV in the spinal cord dorsal horn. The possible functional 

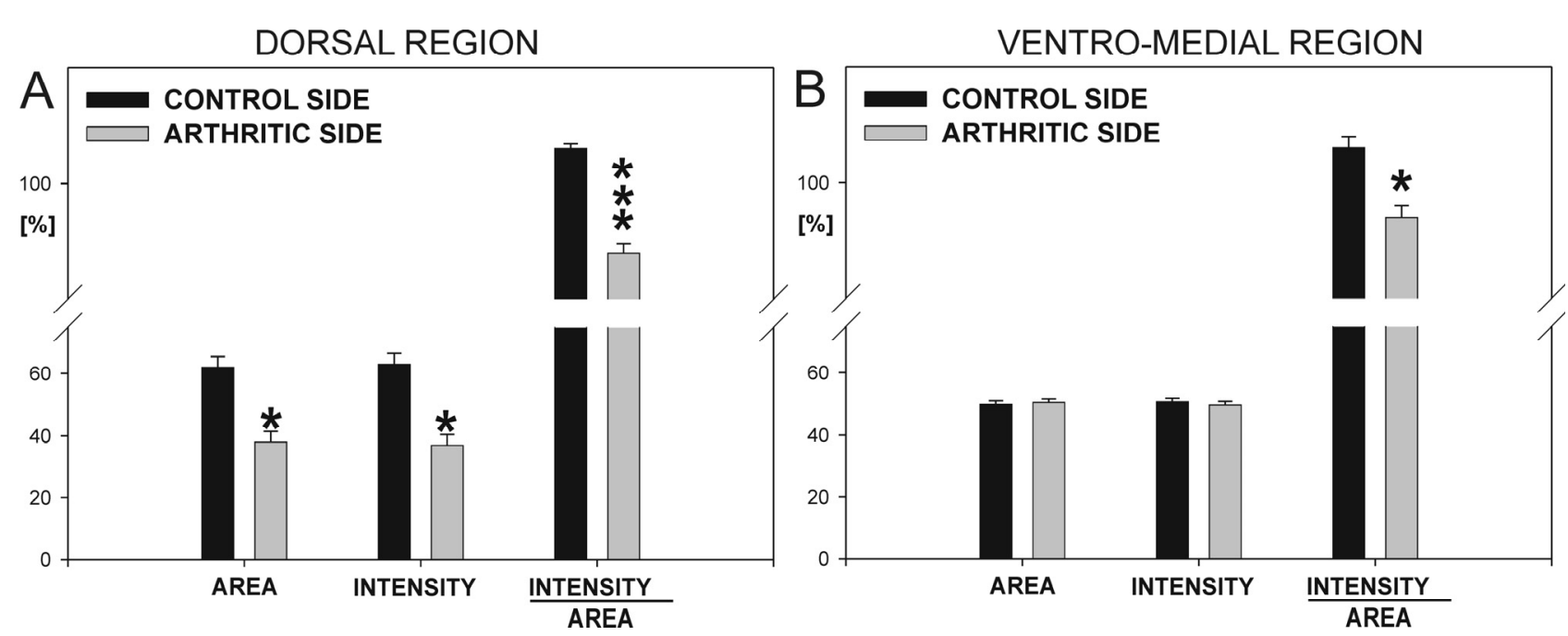

Fig. 4. (A) Densitometric analysis showed that the area of immunopositivity in the symmetrical parts of superficial dorsal horn was smaller on the arthritic side when compared to the contralateral side. The sum of the positive areas on both sides represents $100 \%$. The sum of signal intensity in this area was also lower as well as the quotient of the intensity/area. (B) There was no significant difference in the area and intensity of staining in the ventromedial part of the dorsal horn. However, the quotient of the intensity/area was significantly reduced on the arthritic side. $(* \mathrm{p}<0.05, * * * \mathrm{p}<0.001)$

consequences of this change would be preferentially dependent on its precise morphological location and on the biophysical properties of PV.

It was shown previously that unilateral peripheral inflammation can induce bilateral changes in expression of some molecules at the spinal cord level. However, in most of the studies the effect was much more pronounced on the side ipsilateral to the unilateral inflammation (Traub et al. 1994, Castro-Lopez et al. 1994, Zhang et al. 2002). These changes may also be dependent on the exact model of inflammation used (complete Freund's adjuvant vs. carageenan) and the time when the changes were evaluated after the induction of inflammation. In our experiments we did not observe any obvious changes on the contralateral side when compared to the control animals. In densitometric studies it is usually difficult to compare density of staining in different tissue slices, unless a very significant change in staining is present. Therefore we did not attempt to do densitometric comparisons between the control and experimental animals.

The presence of different calcium-binding proteins such as parvalbumin or calbindin in the spinal dorsal horn was described in detailed morphological studies at light (Yamamoto et al. 1989, Antal et al. 1990, Zhang et al. 1990, Ren and Ruda 1994) as well as electron-microscopic levels (Antal et al. 1990). In general, these calcium-binding proteins, which can act as calcium buffers and have impact on intracellular calcium kinetics, were found to be present in all layers of the

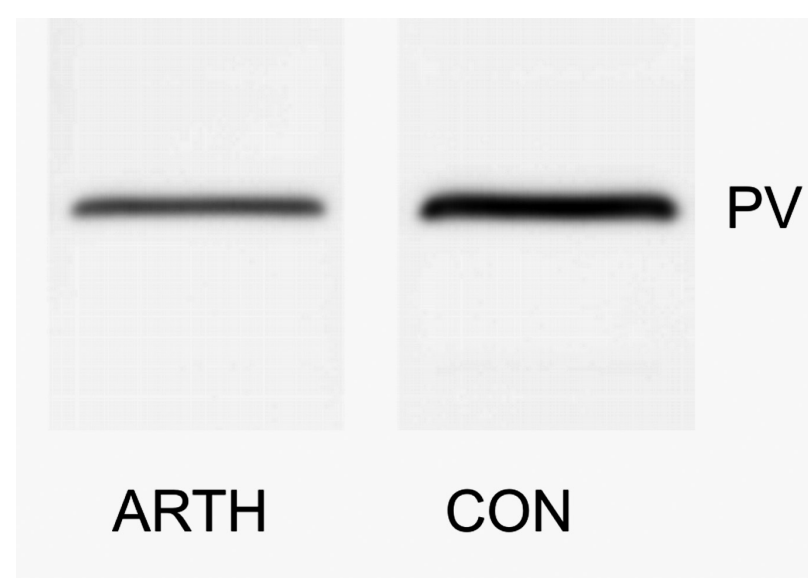

Fig. 5. Example of PV Western blot from ipsilateral (ARTH) and contralateral (CON) spinal cord tissue from animals with unilateral arthritis.

spinal cord. However, the distribution within the spinal cord was different for each of the CBP. Parvalbumin appears to be predominantly expressed in a strongly PV immunopositive band in the inner part of lamina II (Antal et al. 1990, Ren and Ruda 1994), or along the lamina II/III border (Yamamoto et al. 1989), which is in good agreement with the present study. Morphological analysis of the dorsal horn lumbar spinal cord in rats suggested that PV is located mainly in the neuropil near spinal cord PV immunoreactive neurons (Yamamoto et al. 1989). This was further supported by the finding that the intensity of the PV immunoreactive band in the superficial dorsal horn appeared to be unaffected by dorsal rhizotomy or by neonatal capsaicin treatment 
(Yamamoto et al. 1989). It was only slightly reduced after ganglionectomy (Ren and Ruda 1994). Parvalbumin immunoreactivity was found in the postsynaptic as well as in the presynaptic terminals in the inner part of lamina II (Antal et al. 1990), which indicates a possible important role for parvalbumin in modulation of sensory inputs both at presynaptic and postsynaptic levels.

Morphological and functional characterization of neurons in the substantia gelatinosa is quite complex. However, it is clear from numerous studies that neurons in this region are heavily involved in pain transmission and modulation (Willis and Coggeshall 2004). It was shown previously that about $43 \%$ of the neurons in this region are GABA-immunoreactive and are thus presumably GABAergic inhibitory interneurons (Todd and Sullivan 1990). It was suggested that in other areas of the central nervous system PV-containing neurons represent a subpopulation of GABAergic neurons (for review see Baimbridge et al. 1992). At the spinal cord level, it was shown that 5-21\% of the GABAimmunopositive neurons in the substantia gelatinosa region coexpress PV (Antal et al. 1991). Similar results were also shown for a subpopulation of substantia gelatinosa GABAergic neurons expressing green fluorescent protein in transgenic mice (Hantman et al. 2004). However, the population of PV immunopositive neurons in this spinal cord dorsal horn region (laminae II + III) that also show staining for GABA is much higher (75\%, Laing et al. 1994; 60-70\%, Antal et al. 1991). These data suggest that a majority of the PVimmunopositive neurons in this region of the spinal cord dorsal horn may be GABAergic inhibitory interneurons. Based on this evidence it seems plausible to suggest that some of the changes in PV expression in the substantia gelatinosa region seen in our study were due to reduced expression of PV in GABAergic interneurons.

Calcium ions regulate a number of intracellular processes and play a major role in transmembrane signaling in neuronal cells (Baimbridge et al. 1992, Berridge et al. 2003). The role of internal calcium buffers and the temporo-spatial characteristics of intracellular calcium signaling were originally demonstrated in bovine chromaffin cells (Neher and Augustine 1992). PV acts as a mobile calcium buffer with relatively slow binding characteristics and its presence does not reduce the peak of the calcium transient as much as the fast buffers do, but rather accelerates the initial decay of the calcium transient (Chard et al. 1993, Caillard et al. 2000, Lee et al. 2000, Schmidt et al. 2003, Muller et al. 2007). PV presence leads to a fast reduction of calcium concentration and decreased residual calcium concentration after the calcium transient (Atluri and Regehr 1996). The importance of PV for synaptic modulation was shown at the interneuron/Purkinje cell synapse, where elimination of PV from the presynaptic ending changed a depressing synapse into a facilitating one (Caillard et al. 2000, Collin et al. 2005). Similar results were obtained at the Calyx of Held synapse, where presynaptic PV accelerated the decay of the calcium transient and short-term facilitation (Muller et al. 2007). The increased rate of decay of the calcium transient produced by PV was also shown in rat dorsal root ganglion neurons (Chard et al. 1993).

A transient decrease of parvalbumin immunoreactivity was described in cultured cortical neurons, where a time- and dose-dependent reversible decrease in parvalbumin and GAD67 immunoreactivity was present after application of an NMDA receptor antagonist specifically in PV interneurons (Kinney et al. 2006). Marked reduction of PV expression in interneurons was also described in the hippocampus of spontaneously hypertensive rats with stroke, where it correlated with increased immunoreactivity for $\mathrm{PKC}_{\text {gamma }}$ in pyramidal cells (De Jong et al. 1993). $\mathrm{PKC}_{\text {gamma }}$ was shown to be considerably up-regulated under peripheral inflammatory conditions also in lamina II of the lumbar dorsal horn in rats, where it has a close relationship to but no co-expression with PV immunoreactivity (Martin et al. 1999).

In our study we have shown that peripheral inflammation leads to decreased expression of PV in the neuropil of the spinal cord dorsal horn, presumably originating from the processes of GABAergic interneurons. Decreased content of PV in the presynaptic endings of these interneurons could lead to increased residual calcium level during high frequency bursts of activity and potentiate facilitation and increased transmitter release. This would lead to increased potency of inhibitory circuits at the spinal cord level under peripheral inflammatory conditions. While the exact role of PV in the dorsal horn spinal cord neurons needs to be examined further, it seems plausible to suggest that the changes in expression of calcium-binding proteins may play an important role in the modulation of nociceptive transmission.

\section{Conflict of Interest}

There is no conflict of interest. 


\section{Acknowledgements}

The authors thank Mrs. K. Gabová and M. Pytlová for their excellent technical help. Supported by GACR
305/06/1115, GACR 304/08/0256, MSMT CR LC554, MYORES LSH-CT-2004-511978, AV0Z 50110509

\section{References}

ANTAL M, FREUND TF, POLGAR E: Calcium-binding proteins, parvalbumin- and calbindin-D 28k-immunoreactive neurons in the rat spinal cord and dorsal root ganglia: a light and electron microscopic study. J Comp Neurol 295: 467-484, 1990.

ANTAL M, POLGAR E, CHALMERS J, MINSON JB, LLEWELLYN-SMITH I, HEIZMANN CW, SOMOGYI P: Different populations of parvalbumin- and calbindin-D28k-immunoreactive neurons contain GABA and accumulate ${ }^{3} \mathrm{H}$-D-aspartate in the dorsal horn of the rat spinal cord. J Comp Neurol 314: 114-124, 1991.

ATLURI PP, REGEHR WG: Determinants of the time course of facilitation at the granule cell to Purkinje cell synapse. J Neurosci 16: 5661-5671, 1996.

BAIMBRIDGE KG, CELIO MR, ROGERS JH: Calcium-binding proteins in the nervous system. Trends Neurosci 15: 303-308, 1992.

BERRIDGE MJ, BOOTMAN MD, RODERICK HL: Calcium signalling: dynamics, homeostasis and remodelling. Nat Rev Mol Cell Biol 4: 517-529, 2003.

CAILLARD O, MORENO H, SCHWALLER B, LLANO I, CELIO MR, MARTY A: Role of the calcium-binding protein parvalbumin in short-term synaptic plasticity. Proc Natl Acad Sci USA 97: 13372-13377, 2000.

CASTRO-LOPES JM, TAVARES I, TOLLE TR, COIMBRA A: Carrageenan-induced inflammation of the hind foot provokes a rise of GABA-immunoreactive cells in the rat spinal cord that is prevented by peripheral neurectomy or neonatal capsaicin treatment. Pain 56: 193-201, 1994.

CHARD PS, BLEAKMAN D, CHRISTAKOS S, FULLMER CS, MILLER RJ: Calcium buffering properties of calbindin D28k and parvalbumin in rat sensory neurones. J Physiol Lond 472: 341-357, 1993.

COLLIN T, CHAT M, LUCAS MG, MORENO H, RACAY P, SCHWALLER B, MARTY A, LLANO I: Developmental changes in parvalbumin regulate presynaptic $\mathrm{Ca}^{2+}$ signaling. J Neurosci 25: 96-107, 2005.

DE JONG GI, VAN DER ZEE EA, BOHUS B, LUITEN PG: Reversed alterations of hippocampal parvalbumin and protein kinase C-gamma immunoreactivity after stroke in spontaneously hypertensive stroke-prone rats. Stroke 24: 2082-2085, 1993.

HANTMAN AW, VAN DEN POL AN, PERL ER: Morphological and physiological features of a set of spinal substantia gelatinosa neurons defined by green fluorescent protein expression. $J$ Neurosci 24: 836-842, 2004.

KINNEY JW, DAVIS CN, TABAREAN I, CONTI B, BARTFAI T, BEHRENS MM: A specific role for NR2Acontaining NMDA receptors in the maintenance of parvalbumin and GAD67 immunoreactivity in cultured interneurons. J Neurosci 26: 1604-1615, 2006.

LAING I, TODD AJ, HEIZMANN CW, SCHMIDT HH: Subpopulations of GABAergic neurons in laminae I-III of rat spinal dorsal horn defined by coexistence with classical transmitters, peptides, nitric oxide synthase or parvalbumin. Neuroscience 61: 123-132, 1994.

LEE SH, SCHWALLER B, NEHER E: Kinetics of $\mathrm{Ca}^{2+}$ binding to parvalbumin in bovine chromaffin cells: implications for $\left[\mathrm{Ca}^{2+}\right]$ transients of neuronal dendrites. J Physiol Lond 525: 419-432, 2000.

MALENKA RC, NICOLL RA: Long-term potentiation - a decade of progress? Science 285: 1870-1874, 1999.

MARTIN WJ, LIU H, WANG H, MALMBERG AB, BASBAUM AI: Inflammation-induced up-regulation of protein kinase C-gamma immunoreactivity in rat spinal cord correlates with enhanced nociceptive processing. Neuroscience 88: 1267-1274, 1999.

MULLER M, FELMY F, SCHWALLER B, SCHNEGGENBURGER R: Parvalbumin is a mobile presynaptic $\mathrm{Ca}^{2+}$ buffer in the Calyx of Held that accelerates the decay of $\mathrm{Ca}^{2+}$ and short-term facilitation. J Neurosci 27: 22612271, 2007.

NEHER E, AUGUSTINE GJ: Calcium gradients and buffers in bovine chromaffin cells. J Physiol Lond 450: 273-301, 1992. 
PALECEK J, LIPS MB, KELLER BU: Calcium dynamics and buffering in motoneurones of the mouse spinal cord. J Physiol Lond 520: 485-502, 1999.

REN K, RUDA MA: A comparative study of the calcium-binding proteins calbindin-D28K, calretinin, calmodulin and parvalbumin in the rat spinal cord. Brain Res Brain Res Rev 19: 163-179, 1994.

SCHAGGER H, VON JAGOW G: Blue native electrophoresis for isolation of membrane protein complexes in enzymatically active form. Anal Biochem 199: 223-231, 1991.

SCHMIDT H, STIEFEL KM, RACAY P, SCHWALLER B, EILERS J: Mutational analysis of dendritic Ca ${ }^{2+}$ kinetics in rodent Purkinje cells: role of parvalbumin and calbindin D28k. J Physiol Lond 551: 13-32, 2003.

SPICAROVA D, PALECEK J: The role of spinal cord vanilloid (TRPV 1) receptors in pain modulation. Physiol Res $\mathbf{5 7}$ (Suppl 3): S69-S77, 2008.

TODD AJ, SULLIVAN AC: Light microscope study of the coexistence of GABA-like and glycine-like immunoreactivities in the spinal cord of the rat. J Comp Neurol 296: 496-505, 1990.

TOWBIN H, STAEHELIN T, GORDON J: Electrophoretic transfer of proteins from polyacrylamide gels to nitrocellulose sheets: procedure and some applications. Proc Natl Acad Sci USA 76: 4350-4354, 1979.

TRAUB RJ, SOLODKIN A, GEBHART GF: NADPH-diaphorase histochemistry provides evidence for a bilateral, somatotopically inappropriate response to unilateral hindpaw inflammation in the rat. Brain Res 647: 113-123, 1994.

WILLIS WD: Role of neurotransmitters in sensitization of pain responses. Ann N Y Acad Sci 933: 142-156, 2001.

WILLIS WD, COGGESHALL RE: Sensory Mechanisms of the Spinal Cord. Kluwer Academic/Plenum Publisher, New York, 2004.

WOOLF CJ, THOMPSON SW: The induction and maintenance of central sensitization is dependent on N-methyl-Daspartic acid receptor activation; implications for the treatment of post-injury pain hypersensitivity states. Pain 44: 293-299, 1991.

YAMAMOTO T, CARR PA, BAIMBRIDGE KG, NAGY JI: Parvalbumin- and calbindin D28k-immunoreactive neurons in the superficial layers of the spinal cord dorsal horn of rat. Brain Res Bull 23: 493-508, 1989.

ZHANG JH, MORITA Y, HIRONAKA T, EMSON PC, TOHYAMA M: Ontological study of calbindin-D28k-like and parvalbumin-like immunoreactivities in rat spinal cord and dorsal root ganglia. J Comp Neurol 302: 715-728, 1990.

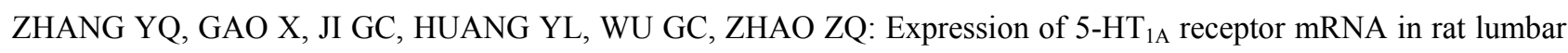
spinal dorsal horn neurons after peripheral inflammation. Pain 98: 287-295, 2002.

ZHOU Z, NEHER E: Mobile and immobile calcium buffers in bovine adrenal chromaffin cells. J Physiol Lond 469: 245-273, 1993. 